\title{
TWEAK and Fn14 expression in the pathogenesis of joint inflammation and bone erosion in rheumatoid arthritis
}

\author{
Anak ASSK Dharmapatni ${ }^{1 *}$, Malcolm D Smith ${ }^{2}$, Tania N Crotti ${ }^{1}$, Christopher A Holding ${ }^{1}$, Cristina Vincent ${ }^{4}$, \\ Helen M Weedon ${ }^{2}$, Andrew CW Zannettino ${ }^{3,6}$, Timothy S Zheng ${ }^{4}$, David M Findlay ${ }^{5,6}$, Gerald J Atkins ${ }^{5,6+}$ and \\ David R Haynes ${ }^{1+}$
}

\begin{abstract}
Introduction: TNF-like weak inducer of apoptosis (TWEAK) has been proposed as a mediator of inflammation and bone erosion in rheumatoid arthritis (RA). This study aimed to investigate TWEAK and TWEAK receptor (Fn14) expression in synovial tissue from patients with active and inactive rheumatoid arthritis (RA), osteoarthritis (OA) and normal controls and assess soluble (s)TWEAK levels in the synovial fluids from patients with active RA and OA. Effects of STWEAK on osteoclasts and osteoblasts were investigated in vitro.

Methods: TWEAK and Fn14 expression were detected in synovial tissues by immunohistochemistry (IHC). Selected tissues were dual labelled with antibodies specific for TWEAK and lineage-selective cell surface markers CD68, Tryptase G, CD22 and CD38. TWEAK mRNA expression was examined in human peripheral blood mononuclear cells (PBMC) sorted on the basis of their expression of CD22. sTWEAK was detected in synovial fluid from OA and RA patients by ELISA. The effect of STWEAK on PBMC and RAW 264.7 osteoclastogenesis was examined. The effect of sTWEAK on cell surface receptor activator of NF Kappa B Ligand (RANKL) expression by human osteoblasts was determined by flow cytometry.
\end{abstract}

Results: TWEAK and Fn14 expression were significantly higher in synovial tissue from all patient groups compared to the synovial tissue from control subjects $(P<0.05)$. TWEAK was significantly higher in active compared with inactive RA tissues $(P<0.05)$. TWEAK expression co-localised with a subset of $C D 38^{+}$plasma cells and with $C D 22^{+}$ B-lymphocytes in RA tissues. Abundant TWEAK mRNA expression was detected in normal human $C D 22^{+} \mathrm{B}$ cells. Higher levels of sTWEAK were observed in synovial fluids isolated from active RA compared with OA patients. sTWEAK did not stimulate osteoclast formation directly from PBMC, however, sTWEAK induced the surface expression of RANKL by human immature, STRO-1 ${ }^{+}$osteoblasts.

Conclusions: The expression of TWEAK by CD22 ${ }^{+} \mathrm{B}$ cells and $\mathrm{CD}_{3} 8^{+}$plasma cells in RA synovium represents a novel potential pathogenic pathway. High levels of sTWEAK in active RA synovial fluid and of TWEAK and Fn14 in active RA tissue, together with the effect of TWEAK to induce osteoblastic RANKL expression, is consistent with TWEAK/Fn14 signalling being important in the pathogenesis of inflammation and bone erosion in RA.

\footnotetext{
* Correspondence: a.dharmapatni@adelaide.edu.au

† Contributed equally

'Discipline of Anatomy and Pathology, School of Medical Sciences,

University of Adelaide, Frome Road, Adelaide, SA 5005, Australia

Full list of author information is available at the end of the article
} 


\section{Introduction}

TWEAK (TNF-like weak inducer of apoptosis) is a recently described member of the TNF superfamily. It is reported to exert a variety of biological effects through ligation with its receptor, Fn14. The biological effects of TWEAK include induction of pro-inflammatory cytokines, modulation of the immune response and angiogenesis, stimulation of apoptosis and regulation of tissue repair and regeneration $[1,2]$. The pro-inflammatory effects of TWEAK/Fn14 signalling are mediated by several signalling cascades, including NF-B and the mitogen-activated protein kinases (MAPK), ERK1/2, JNK1/2 and p38 [3]. TWEAK induces the production of a large number of pro-inflammatory molecules, such as matrix metalloproteinase (MMP1), IL-6, IL-8, MCP-I and Regulated upon Activation Normal $\mathrm{T}$ Cell Expressed and Secreted (RANTES) by synoviocytes and fibroblasts, as well as ICAM-1, E-selectin, IL-8, and MCP-1 by endothelial cells [4]. The majority of these cytokines are induced by TWEAK/Fn14 induction of the NF- $\kappa \beta$ signalling pathway $[3,5]$. The pro-inflammatory effects of TWEAK are seen in various cell types including glomerular mesangial cells [6], human umbilical vein endothelial cells (HUVEC) [7], human gingival fibroblasts [8], human dermal fibroblasts, synoviocytes [9], chondrocytes, and fibroblasts [2].

Recent reports from us [10] and others [11] are consistent with TWEAK being a key mediator of joint pathology in murine RA models and in human RA $[12,13]$. Specifically, recombinant TWEAK enhanced the production of MCP-1 and MIP-2 by synovial cells from collagen induced arthritis (CIA) mice in vitro, while the addition of TWEAK monoclonal antibody ameliorated paw swelling, synovial proliferation and inflammatory cell accumulation in CIA $[10,11]$. A role for TWEAK has been described in human RA, where TWEAK induced the proliferation of synovial fibroblasts and increased the production of inflammatory cytokines and chemokines, as well as the expression of ICAM-1 [12]. High serum levels of TWEAK, TNF- $\alpha$ and IL- 6 were seen in RA patients as compared to normal controls [13]. Moreover, serum TWEAK levels correlated with the disease activity score (DAS28) in RA patients and high serum TWEAK levels demonstrated a correlation with short-term response to etanercept treatment [13]. Higher levels of TWEAK were found in RA compared to psoriatic synovium [14]. In the current study we examine TWEAK expression in a larger group of patient-derived samples that encompassed active and inactive RA, osteoarthritic (OA) and normal patients. In addition, levels of soluble (s) TWEAK in the synovial fluids of active RA compared with OA patients were determined.

Pertinent to the pathogenesis of cartilage and bone loss in RA, TWEAK has been demonstrated to promote bone and cartilage destruction through inhibition of chondrogenesis, osteogenesis and the induced production of matrix metalloproteinase (MMP)-3 [10,15]. We have recently described a role for TWEAK in human osteoblast differentiation [16] and Polek et al. have proposed its role in osteoclastogenesis [17]. In a more recent study, we demonstrated a significant relationship between serum TWEAK levels and bone erosion markers in patients with bone destructive multiple myeloma [18]. In addition, we have shown that TWEAK, alone and together with TNF- $\alpha$, induces the expression by osteoblasts and osteocytes, in vitro and in ex vivo bone, of the bone formation inhibitor, sclerostin, via a MAPKdependent pathway [16] suggesting a means by which TWEAK may inhibit bone formation during inflammatory bone remodeling. These findings suggest that TWEAK may modulate the bone erosion associated with several diseases, such as rheumatoid arthritis (RA) and multiple myeloma. The present study extends these findings by investigating the effect of sTWEAK on osteoclastogenesis and furthermore the effect of sTWEAK on osteoblasts in vitro.

\section{Materials and methods \\ Patients}

This study was approved by the Human Ethics Committees of the University of Adelaide and The Repatriation General Hospital, and informed consent was obtained from all patients and healthy donors. RA patients fulfilled the 1987 revised criteria of the American College of Rheumatology (ACR) [19]. OA patients fulfilled the criteria by Altman and colleagues [20]. All patients with active RA had joint inflammation and patients with inactive RA were in remission after successful disease modifying anti-rheumatic drug (DMARD) treatment. Normal synovial tissues were obtained as previously described [21]. Synovial tissue was obtained from 39 patients (10 active RA, 9 inactive RA, 10 OA patients and 10 normal subjects) at the time of knee arthroscopy or total knee replacement surgery (OA patients) at the Rheumatology Unit, Repatriation General Hospital, South Australia. Characteristics of patients for IHC are summarised in Table 1.

Synovial fluid samples for ELISA were obtained from 17 active RA (5 female/12 male) and 16 OA (7 female/9 male) subjects. The mean age $( \pm$ SEM) of the OA group was $66.40 \pm 3.18$ and of the active RA group was $63.19 \pm 3.78$.

\section{Immunohistochemical detection of TWEAK}

TWEAK was detected using the previously described IHC method [22,23]. Briefly, tissue sections $(5 \mu \mathrm{m})$ were deparaffinised, pre-treated with proteinase-K to unmask the antigen and treated with sodium azide in PBS $(0.1 \%$ 
Table 1 Demographic of patient for IHC TWEAK and Fn14 in synovial tissues

\begin{tabular}{|c|c|c|c|c|}
\hline & Control & Active RA & Inactive RA & $O A$ \\
\hline Gender (Female/Male) & $4 / 6$ & $6 / 4$ & $3 / 6$ & $3 / 7$ \\
\hline Mean Age (years) (range) & 40.2 (25 to 58$)$ & 69.2 (31 to 86$)$ & 72.33 (60 to 79$)$ & 69.3 (55 to 77 ) \\
\hline Disease duration (months) (range) & NA & 3.1 (2 to 6) & 21.77 (7 to 36$)$ & NA \\
\hline Rheumatoid Factor & NA & $6 / 10$ & $5 / 9$ & NA \\
\hline Erosions & NA & $2 / 10$ & $2 / 9$ & NA \\
\hline C-Reactive protein median $(\mathrm{mg} / \mathrm{ml})$ (range) & NA & 67.5 (27.0 to 307.0$)$ & $6(2.0$ to 26.0$)$ & NA \\
\hline \multirow[t]{4}{*}{ Treatment } & NA & 9 NSAIDs & 5 im gold & 6 NSAIDs \\
\hline & & 1 prednisolone & 2 methotrexate & 3 NSAIDs \\
\hline & & & 1sulphasalazine & 1 panadeine \\
\hline & & & 1 plaquenil & \\
\hline
\end{tabular}

NA, not available; NSAIDs: non steroidal anti-inflammatory drugs.

w/v) and $\mathrm{H}_{2} \mathrm{O}_{2}(0.3 \% \mathrm{v} / \mathrm{v})$ to inhibit endogenous peroxidase. Sections were then incubated overnight with mouse anti-human TWEAK monoclonal antibody (MAb), P2D10 (15 $\mu \mathrm{g} / \mathrm{ml})$ [6]. Secondary antibody (HRP-conjugated goat anti-mouse IgG, DAKO, Botany, NSW, Australia) was then added, followed by HRPconjugated swine anti-goat IgG (Biosource, Camarillo, CA, USA). The colour reaction was developed using AEC (3,9 aminoethylcarbazole from Sigma, St. Louis, MO, USA). Counterstaining was performed using Harris haematoxylin and lithium carbonate. An isotypematched, non-binding control antibody (1D4.5, isotype IgG2a) at an identical IgG concentration or omission of the primary antibody, was used as a negative control. Tonsil tissues obtained at surgery were used as controls for lymphocytic staining.

\section{Immunohistochemical detection of Fn14}

Fn14 was detected using previously published methods [24]. Briefly, sections were deparaffinised and pre-treated with $10 \mathrm{mM}$ sodium citrate buffer $\left(\mathrm{pH} \mathrm{6.0)}\right.$ at $95^{\circ} \mathrm{C}$ for 20 minutes. Endogenous peroxidase was then blocked using $0.3 \% \mathrm{v} / \mathrm{v} \mathrm{H}_{2} \mathrm{O}_{2}$ in methanol solution. Blocking serum was applied, according to the manufacturer's instructions (Vectastain Universal Elite ABC kit, Vector Laboratories, Burlingame, CA, USA). Sections were then incubated with anti-human Fn14 MAb (ITEM1; Biolegend, San Diego, CA, USA) at $20 \mu \mathrm{g} / \mathrm{ml}$ overnight. Appropriate biotinylated secondary antibody was then added in the presence of $10 \%$ normal horse serum. Sections were then treated with avidin-biotin complex reagent before being stained with 3,3' Diaminobenzidine (DAB) (Vector Labs). Counter-staining and isotypematched negative controls were performed as above for TWEAK staining.

\section{Dual immunohistochemistry}

Double staining was performed to identify specific cell types expressing TWEAK using a previously published method [23]. Anti-TWEAK antibody was combined with MAbs for human cell surface markers: CD68 (macrophage; clone KP-1, Dako), CD22 (B lymphocyte; MAB1968, R\&D Systems, Minneapolis, MN, USA), Tryptase G3 (mast cell; Cell Marque, Rocklin, CA, USA) and CD38 (plasma cells, BD Biosciences, Franklin Lakes, NJ, USA). After the single immunoperoxidase staining described above, the colour reaction was developed using an alkaline phosphatase reaction. Normal donkey serum (20\% in PBS) was used to block non-specific binding, followed by incubation with cell surface marker antibodies overnight. Sections were incubated with APconjugated donkey anti-mouse IgG (Jackson Immuno Research, West Grove, PA, USA) as secondary antibody followed by incubation with mouse APAAP IgG (Dako, Botany, NSW, Australia). Colour was developed using a Fast Blue substrate. Using this method, immunoperoxidase stained cells were red, immuno-alkaline phosphatase stained cells were blue, while cells with co-expression were purple.

\section{Quantification of immunohistochemical staining}

A semi-quantitative (SQA) scoring method was used to assess single immunohistochemical labelling, using a validated scoring system [22]. SQAs were analysed statistically by SPSS 11.5 software (SPSS Inc. Chicago, IL, USA) using non-parametric analysis. A $P$-value of less than 0.05 was considered to be significant.

\section{Detection of TWEAK mRNA in CD22 ${ }^{+}$B Cells}

Human peripheral blood mononuclear cells (PBMC) were prepared from normal buffy coat blood packs (Australian Red Cross Society, Adelaide, SA) on Ficoll gradients, as previously described [25]. CD22-expressing cells were isolated by fluorescence activated cell sorting (FACS) after staining with a mouse anti-human CD22 monoclonal antibody directly conjugated to phycoerythrin (anti-human CD22RPE, Clone 4KB128, Dako, Golstrup, Denmark), by a method essentially described 
previously [25]. CD22 ${ }^{+}$or $\mathrm{CD} 22^{-}$cells were pelleted by centrifugation and resuspended in Trizol reagent. Total RNA and complementary DNA (cDNA) were prepared, and real-time reverse transcription polymerase chain reaction (RT-PCR) performed for the expression of TWEAK mRNA, as previously described [16].

\section{Detection of sTWEAK in synovial fluids}

sTWEAK levels were measured following the manufacturer's instructions using a TWEAK instant ELISA kit (Bender MedSystem, Burlingame, CA, USA). Briefly, samples were added in duplicate to a 96-microwell plate, which had been pre-coated with antibody, capture antibody and secondary antibody and colour was developed using tetramethylbenzidine (TMB) substrate. The absorbance was read at $450 \mathrm{~nm}$ using Multiskan Ascent plate reader (Thermo Labsystems, Helsinki, Finland). A standard curve was generated from the control sera provided with the kit. The difference between the mean levels of sTWEAK in the two groups was analysed using an independent sample $t$ test. A $P$-value of less than 0.05 was considered to be significant.

\section{Osteoclastogenesis assays in human PBMC and RAW 264.7 cells}

Human CD $14^{+}$PBMC were isolated by FACS, as described previously in detail [25]. The isolated cells were plated at $2 \times 10^{5}$ cells/well into a 96-microwell plate for tartrate resistant acid phosphatase (TRAP) staining or on whale dentine slices for resorption assays. Cells were cultured in $\alpha$ MEM medium containing $10 \%$ foetal calf serum (FCS) and $10 \mathrm{nM}$ dexamethasone (Fauldings, Adelaide, SA, Australia) and rhM-CSF (25 ng/ml; Millipore, Temecula, CA, USA). Cultures received additional rhTWEAK and/or rhRANKL as indicated and were fed every three days. TRAP activity was assessed at 9 days and resorption was assessed by scanning electron microscopy (SEM) after 14 days, as described previously [25].

Murine RAW 264.7 cells were plated at $1 \times 10^{5}$ cells/ well into 96-well plates, in the presence or absence of recombinant human (rh) Receptor Activator of Nuclear factor Kappa-B Ligand (RANKL) (100 ng/ml) (Millipore) and/or rhTWEAK (10 to $800 \mathrm{ng} / \mathrm{ml}$ ). Cells were cultured for up to seven days in $\alpha$-MEM medium containing $10 \%$ FCS and $10 \mathrm{nM} 1 \alpha, 25$-dihydroxyvitamin $\mathrm{D}_{3}$ $(1,25 \mathrm{D})$ [26] then stained for TRAP using a commercial kit (Sigma) at Day 7.

\section{Osteoblast assays}

Human primary osteoblasts were cultured from cancellous bone samples obtained from patients undergoing total hip replacement surgery, as described previously [16]. Cells $\left(10^{5} /\right.$ well) were seeded into wells of a six-well plate and cultured overnight in $\alpha$ MEM medium containing $10 \% \mathrm{v} / \mathrm{v}$
FCS. Cells were then cultured untreated or treated with recombinant human TWEAK $(50 \mathrm{ng} / \mathrm{ml})$ for three days [16]. Cells were enzymically removed and stained for STRO-1 [27] as previously described [28], and RANKL (MAB6261, R\&D Systems) using an anti-mouse IgM-PE and anti-mouse IgG-FITC conjugate, respectively. Isotypematched negative control antibodies 1A6.12 (IgM) and $1 \mathrm{~B} 5\left(\mathrm{IgG}_{1}\right)$ were used to determine the level of background fluorescence for each fluorochrome and the compensation settings. Stained cells were analysed on a FACStar $^{\text {PLUS }}$ flow cytometer (Becton Dickinson, Sunnyvale, CA, USA). The percentages of cells positive for either or both STRO-1 and RANKL were calculated.

\section{Results}

Immunohistochemistry

Immunohistochemical staining demonstrated that TWEAK was expressed at significantly higher levels in synovial tissue from active RA, inactive RA and OA patients (Figure $1 \mathrm{~A}-\mathrm{C}$, respectively) compared to normal

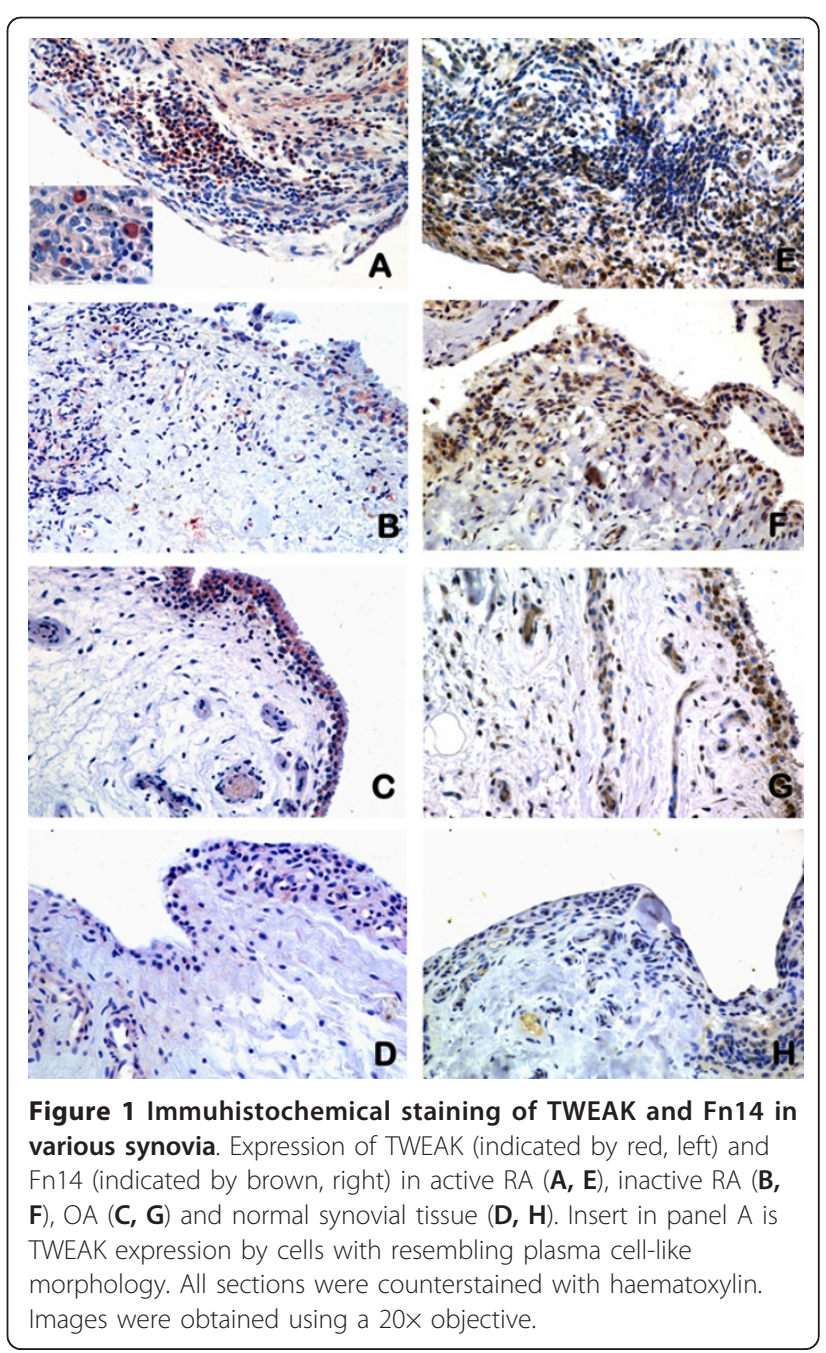


controls (Figure 1D). The majority of cells expressing cytoplasmic TWEAK had either a macrophage-like morphology and were scattered in the synovial lining or appeared to be lymphocytes in the sub-lining region of active RA specimens. Significantly higher levels of TWEAK staining were observed in active RA compared to inactive RA synovial tissue $(P<0.05$, Figures $1 \mathrm{~A}, \mathrm{~B}$ and 2). In OA tissues (Figure $1 C$ ), TWEAK expression was confined mainly to the lining region with much weaker staining intensity compared to active RA synovial tissue. In addition, TWEAK was weakly expressed by cells lining the blood vessels, particularly in the synovial tissue from normal controls (Figure 1D).

Fn14 was highly expressed in synovia from active RA patients (Figure 1E) compared to other groups (Figure $1 \mathrm{~F}-\mathrm{H})$. There was a statistically significant difference between Fn14 expression in normal tissue compared to diseased groups (Figure 2), but not between active RA and inactive RA groups. There was a strong correlation between the relative expression of TWEAK and Fn14 in synovial tissues (Kendall tau_ b test, $r=0.443$, $P=0.001)$.

\section{Cell types expressing TWEAK and Fn14}

In the active RA tissues a subset of the CD68-positive cells (macrophages) weakly expressed TWEAK (Figure $3 \mathrm{~A})$. In addition there is a subset of TWEAK positive cells which are positive for the plasma cell marker, CD38 (Figure 3B). However, the majority of cells strongly expressing TWEAK were positive for the B lymphocyte marker, CD22 (Figure 3C, D). TWEAK was not expressed by cells staining positive for the mast cell marker, Tryptase G, in any of the patient tissues (data not shown). On close inspection, some of the cells expressing TWEAK contained multiple nuclei (arrowed in Figure 3E). In the control tonsil tissue TWEAK was expressed by only a few B lymphocytes (Figure 3F). Due to the staining conditions required for the Fn14 MAb, dual labelling studies with this antibody were not possible. The majority of cells expressing Fn14 were similar in morphology to those expressing TWEAK (monocytes or lymphocytes). Close examination of the cells staining for Fn14 showed that some of these cells were also multinucleated cells (Figure 3G). Cells lining the small blood vessels also expressed Fn14. This was seen to some extent in all the tissues but was particularly prominent in the active RA tissue (Figure $3 \mathrm{H}$ ).

Given that the clone used to detect TWEAK, P2D10, is function neutralising [6], it is unlikely that TWEAKpositivity was due to Fn14-positive cells binding soluble TWEAK. However, to confirm that B cells are a potential source of TWEAK, CD22-expressing or nonexpressing cells were isolated by FACS and real-time RT-PCR performed for TWEAK mRNA expression. As shown in Figure 4, CD $22^{+}$PBMC from two healthy volunteers expressed abundant TWEAK mRNA, relative to that of the housekeeping gene, GAPDH, and did so to a greater extent than the CD22- fraction, consisting largely of T-cells and monocytes.

\section{TWEAK in synovial fluids from RA and OA}

While not statistically significant, there was a trend $(P=$ 0.079) for higher measurable levels of TWEAK protein in active RA synovial fluid $(1,226 \pm 235 \mathrm{pg} / \mathrm{ml})$ than in the OA samples $(713 \pm 134 \mathrm{pg} / \mathrm{ml})$ (Figure 5).

\section{Effect of soluble TWEAK on osteoclastogenesis in vitro}

Recombinant human (rh) TWEAK at concentrations as high as $800 \mathrm{ng} / \mathrm{ml}$ in the presence of M-CSF did not stimulate osteoclast differentiation from unfractionated

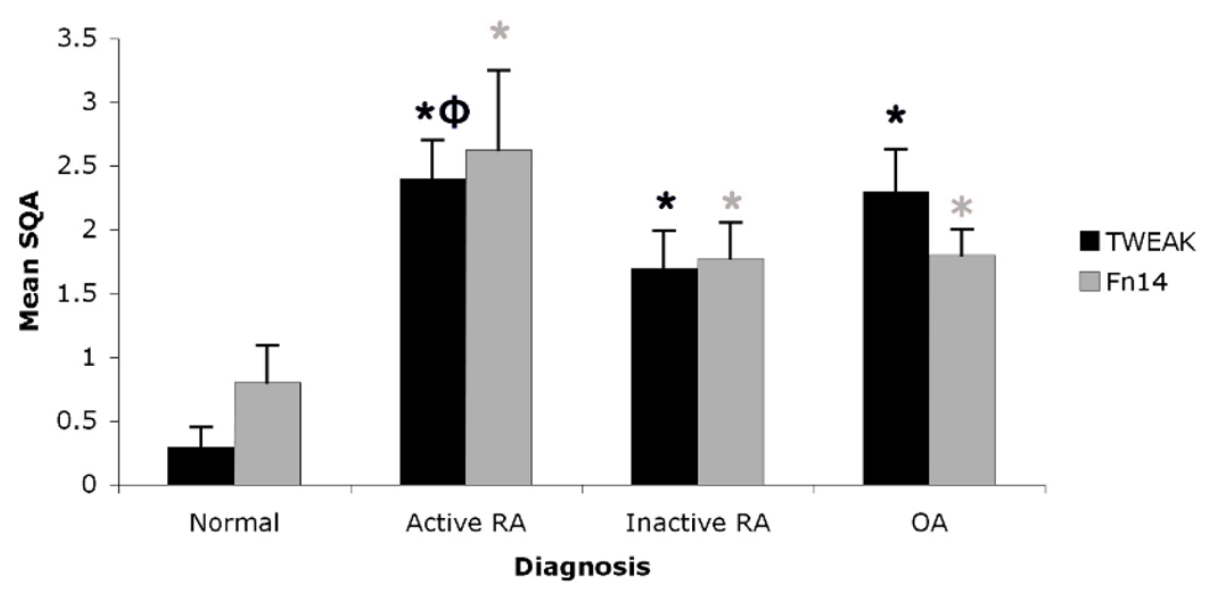

Figure 2 SQA analysis of TWEAK and Fn14. TWEAK and Fn14 expression in various synovial tissues as expressed by mean SQA \pm SEM. Significant differences $(P<0.05)$ are indicated by * compared to normal and $\Phi$ compared to inactive RA synovial tissue. 


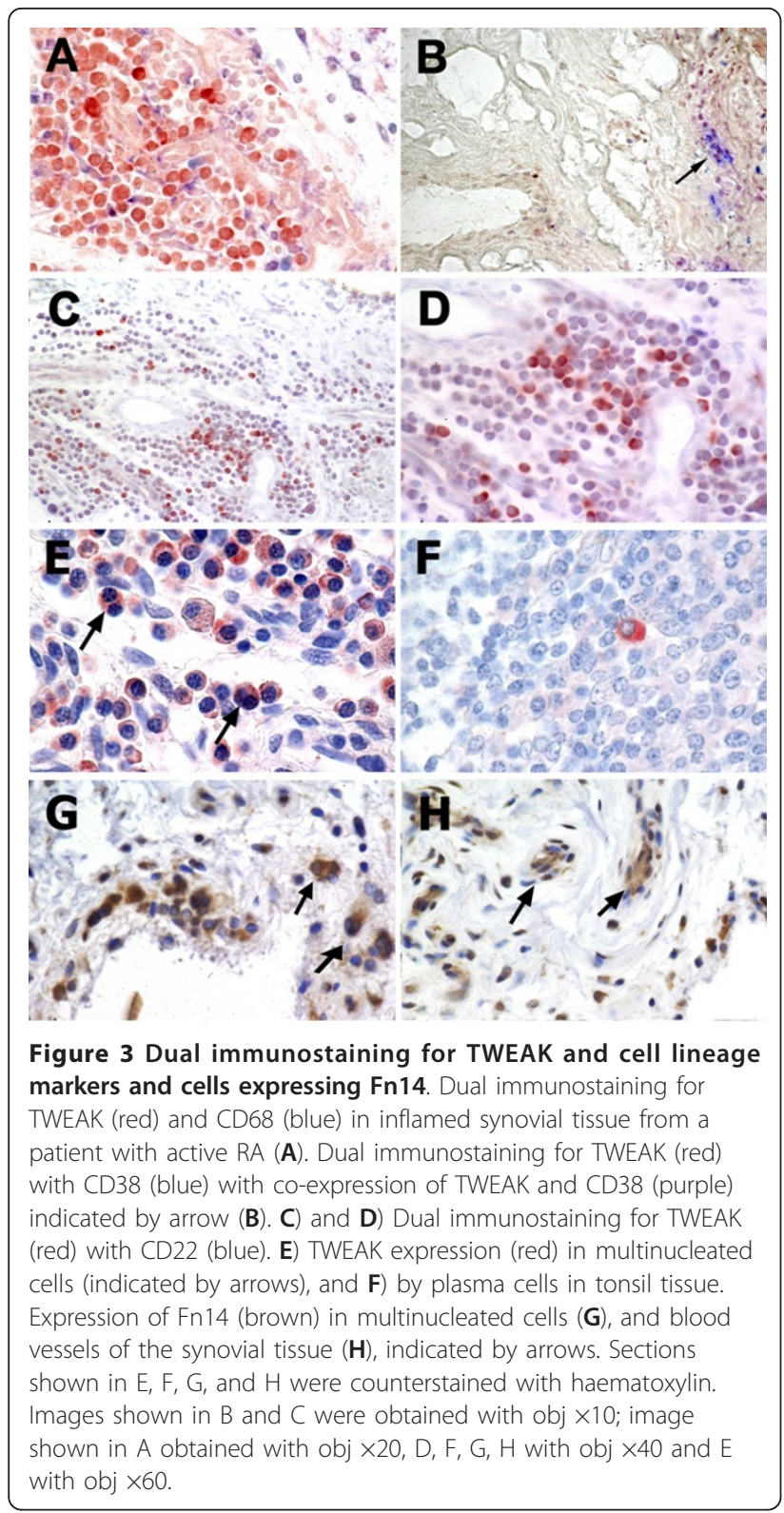

PBMC (Figure 6) or from fractionated $\mathrm{CD} 14^{+}$human PBMC (not shown). Furthermore, RANKL/M-CSF osteoclastogenesis was inhibited rather than stimulated by rhTWEAK (Figure 6) as assessed by both TRAP staining and resorption pit formation on dentine slices (Figure 6). Identical results were obtained when murine RAW 264.7 cells were used as osteoclast precursors (data not shown).

\section{Effects of soluble TWEAK on cell surface RANKL expression}

Treatment of human primary osteoblasts with rhTWEAK for three days resulted in the increased cell

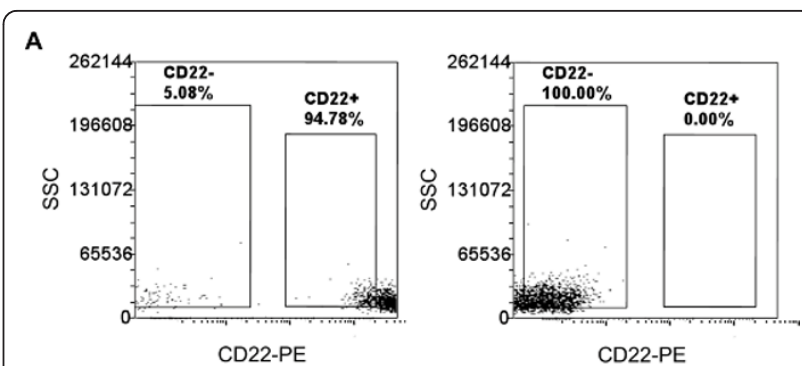

B

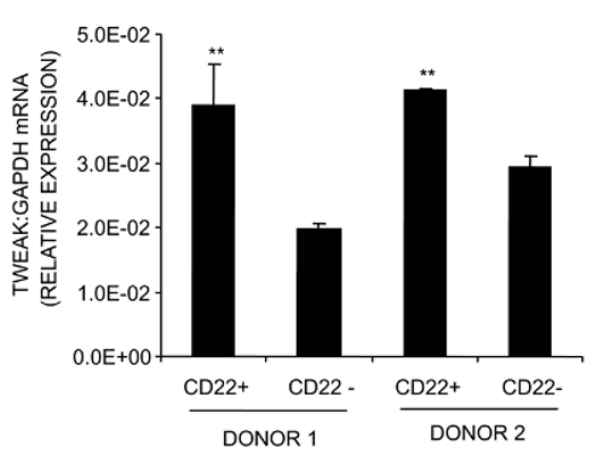

Figure 4 TWEAK expression by PBMC. PBMC from two healthy volunteers were sorted by FACS based on their expression of CD22, yielding $\mathrm{CD}_{22}{ }^{+}$and $\mathrm{CD} 22^{-}$populations of greater than $94 \%$ purity based on post-sort analysis (A). Isolated cells were then analysed for TWEAK mRNA expression relative to that of GAPDH, by real-time RT$P C R(B)$. Data shown are means of triplicate reactions \pm SD. Differences in relative expression of TWEAK mRNA between $C D 22^{+}$ and CD22- populations were tested by Student's $t$-test ( ${ }^{* *} P<0.001$ ).

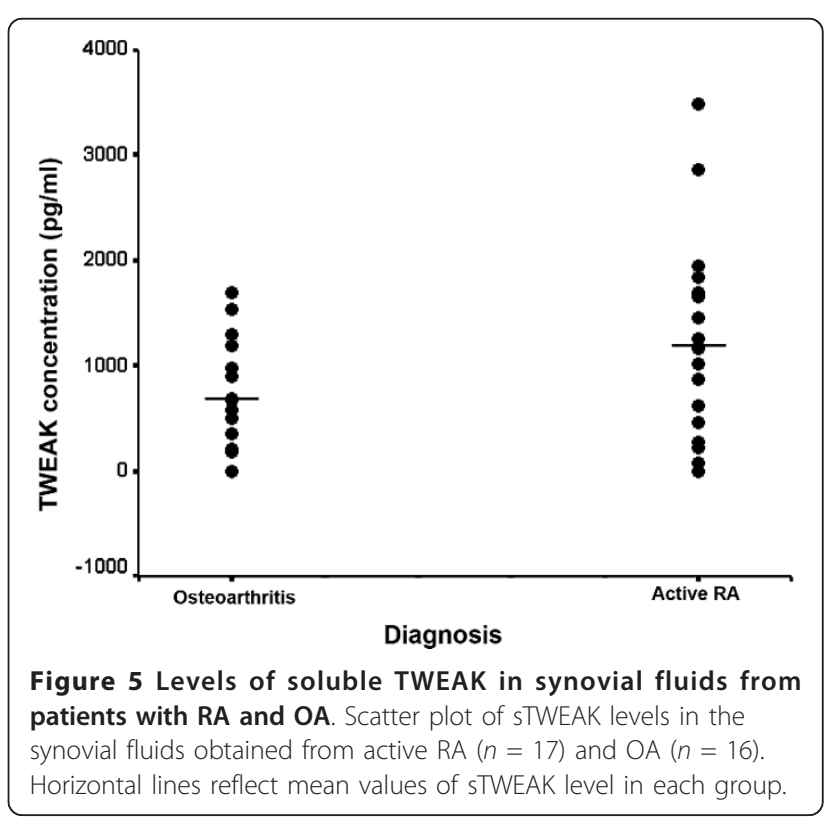


surface expression of RANKL. Furthermore, RANKL expression was induced on a population of osteoblasts expressing the immature osteoblast marker, STRO-1 (Figure 7). Together, these findings are consistent with the induction by TWEAK of a pro-osteoclastogenic osteoblastic phenotype.

\section{Discussion}

The findings of this study are consistent with the growing evidence that TWEAK is a mediator of the joint destruction both in animal models of RA $[10,11]$ and in human RA [12]. Recently, a number of cell types involved in the pathogenesis of RA have been reported to express TWEAK and its receptor, Fn14. While synovial fibroblasts from human patients with active RA have been reported to express TWEAK and Fn14 [14] another report demonstrated TWEAK expression by an unidentified CD45-positive haemopoietic cell population and Fn14 expression by both CD45-positive and -negative cells [12]. In contrast, van Kuijk and co-workers [14] reported TWEAK/Fn14 expression by macrophages but not by lymphocytes. The current study demonstrates that several cell types in RA synovial tissue express TWEAK, including CD68-positive macrophages, confirming the results of van Kuijk and co-workers [14]. Interestingly and in contrast to van Kuijk and co-workers, we observed that a sub-population of CD22-positive B lymphocytes and CD-38 positive plasma cells found in lymphocyte aggregates in the sub-lining synovial tissues expressed TWEAK. Our findings are consistent with those of Kraan and coworkers who found that the numbers of $\mathrm{CD} 38^{+}$plasma cells and $\mathrm{CD} 22^{+} \mathrm{B}$ cells in RA were the best discriminating markers when comparing RA to non-RA inflammatory synovial samples [22].

In the current study, TWEAK expression by plasma cells is in agreement with our recent finding that demonstrated the expression of TWEAK in bone marrow plasma cells in patients with multiple myeloma [18]. In that study, serum levels of TWEAK correlated with the serum bone resorption marker, $\beta$-crosslaps, and levels of the osteoclast recruitment chemokine, CXCL12, strongly implying a pathological role for plasma cell-derived TWEAK in bone erosion associated with multiple myeloma [18]. In the current study, we found that the TWEAK-positive B-Lymphocytes in the germinal centres of normal tonsil sections were rare, consistent with the infrequency of $\mathrm{CD} 22^{+} \mathrm{B}$ cells at this site [29]. While the CD22 ${ }^{+}$TWEAK $^{+}$expressing B cells may be a specific population associated with the chronic inflammation seen in RA, CD22-positive B lymphocytes isolated from human normal peripheral blood also expressed abundant levels of TWEAK mRNA, suggesting that B-lineage cells are a source of TWEAK. Based on our findings in multiple myeloma [18] these cells 


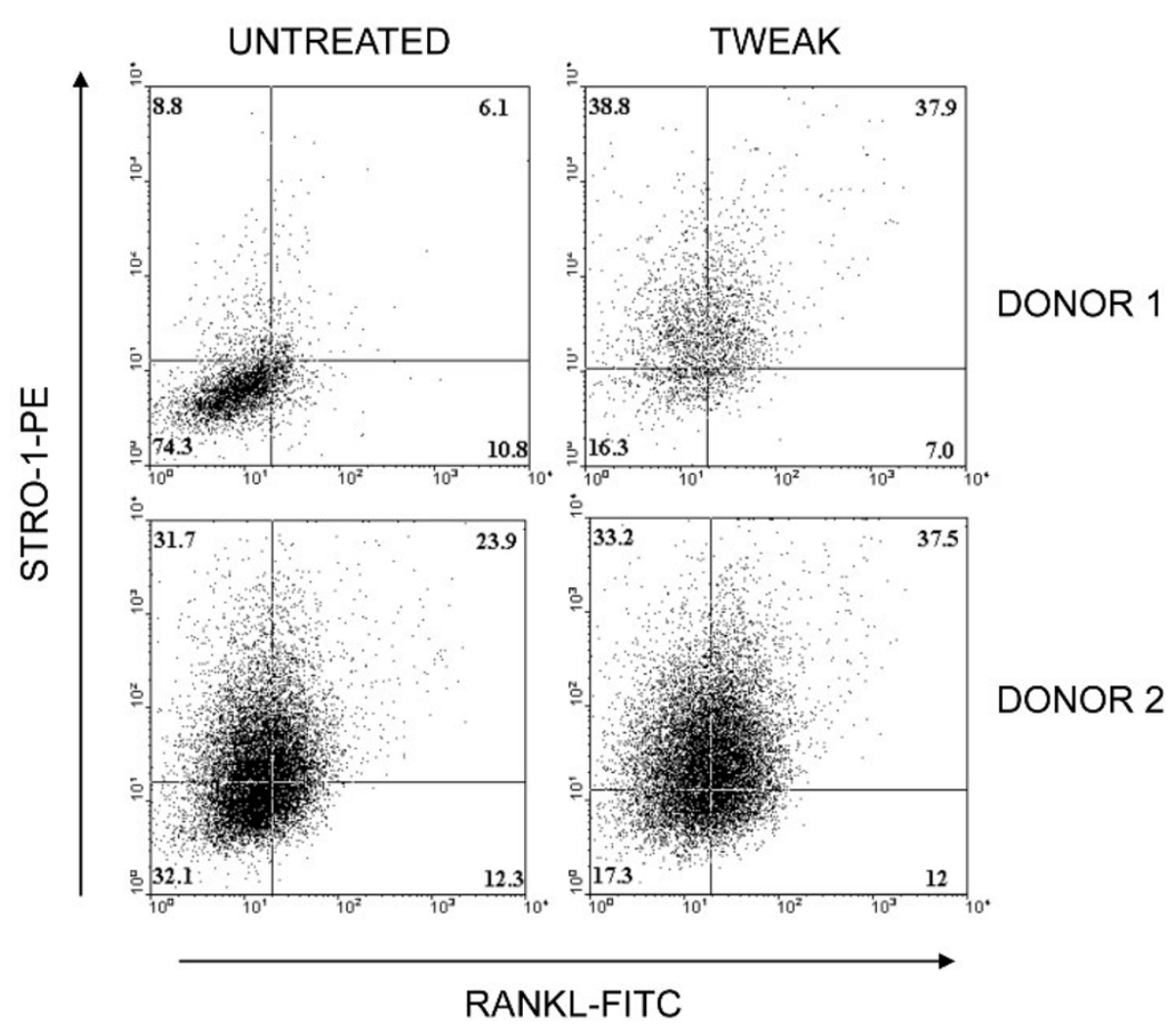

Figure 7 Effect of soluble TWEAK on cell surface RANKL expression by STRO-1 osteoblast subpopulation. Human primary osteoblasts were cultured untreated or treated with rhTWEAK $(50 \mathrm{ng} / \mathrm{ml}$ ) for three days, harvested by collagenase/dispase digestion and stained for STRO-1 and RANKL expression. Stained cells were analysed by FACS. The percentage of cells present in each of the four sub-populations is indicated.

Data are presented for two independent donors' cells.

may possibly also be associated with the bone erosion characteristic of RA. Interestingly, an immunotherapeutic approach that targets CD22, epratuzumab, has proved efficacious in the treatment of non-Hodgkin lymphoma, and in the autoimmune diseases, systemic lupus erythematosus and primary Sjögren's syndrome [30]. Our findings suggest that CD22 and CD38 are also potential therapeutic targets in RA.

We were unable to verify the cell types expressing Fn14 using dual label IHC for technical reasons; however, many of these cells also had the morphology of lymphocytes and monocytes and importantly our observation indicates that Fn14 was strongly expressed by multinucleated cells within the synovial tissue. We have previously demonstrated that these multinucleated cells were preosteoclasts [31]. It is well known that the active rheumatic joint contains an influx of osteoclast precursors [31]. This is also consistent with a recent study by our group demonstrating the expression of TWEAK and Fn14 by multinucleated cells in gingival tissues from chronic periodontitis patients [32]. This would suggest that TWEAK-Fn14 signalling may stimulate osteoclastogenesis and hence could contri- bute to the bone loss associated with chronic inflammatory diseases $[10,11]$.

The observation that Fn14 is expressed by cells associated with blood vessels is consistent with the report that TWEAK regulates human endothelial cell proliferation, migration and survival in vitro $[33,34]$. Both TWEAK and Fn14 expression was associated with synovial tissue blood vessels suggesting that TWEAK has an autocrine effect on the angiogenesis seen in RA synovial tissue. Furthermore, this is consistent with the perivascular expression of TWEAK and Fn14 expression in RA and psoriatic synovial tissue [14].

A significant decrease in serum TWEAK has been reported in RA patients responding to treatment with etanercept $[13,14]$ while persistent TWEAK/Fn14 expression was seen in a RA patient cohort receiving infliximab treatment [14] suggesting a role for TWEAK in modulation of disease activity. Our data demonstrated significantly lower expression of TWEAK in the inactive RA synovial tissues. In the majority of patients this followed successful non-TNF targeted DMARD treatment, suggesting that DMARDs may reduce TWEAK 
expression as seen in the etanercept-treated patients. Higher TWEAK expression in active RA was also reflected in the increased synovial fluid levels of sTWEAK compared with those in OA in our study. Based on this, we suggest that monitoring the sTWEAK levels in synovial fluids could possibly be another marker of disease progression.

It was previously reported that TWEAK stimulates the differentiation of RAW 264.7 monocyte/macrophage cells into functional osteoclasts, and did so in the absence of Fn14 expression [17]. However, we could not reproduce these findings in vitro using recombinant TWEAK to directly stimulate osteoclast formation from RAW 264.7 cells, or from human PBMC, either unfractionated or sorted on the basis of CD14 expression, a fraction shown previously to contain the osteoclast precursor $[25,35]$. Although the present study demonstrated that multinucleated cells expressed Fn14, our data indicate that the stimulation of osteoclast formation by TWEAK may not be due to a direct interaction with osteoclast precursors. However, we have demonstrated that stromal osteoblasts express Fn14 and are sensitive to the effects of TWEAK $[10,16]$, including the induced expression of RANKL and CXCL12 mRNAs [18]. This supports an indirect role for TWEAK in recruiting and generating osteoclasts, via the osteoblast. In the current study we confirmed that TWEAK induced the expression of cell surface RANKL protein and did so in a population of human osteoblasts expressing the immature osteoblast cell surface marker, STRO-1, cells we have previously demonstrated are capable of supporting osteoclastogenesis [36]. This is reminiscent of our earlier study, in which RANKL mRNA expression was preferentially induced in STRO-1-positive osteoblasts in response to $1 \alpha, 25$-dihydroxyvitamin $\mathrm{D}_{3}$ [37]. In addition, we recently reported a novel role for TWEAK in the regulation of osteoblast differentiation [16] and showed that TWEAK, and the combination of TWEAK and TNF $\alpha$, induced the expression of sclerostin, a negative regulator of bone formation [38], and as we recently demonstrated [39], human osteoblast differentiation and matrix mineralization. Thus, the elevated TWEAK expression we see in RA may not only stimulate bone resorption by osteoclasts by induction of RANKL on osteoblasts but also inhibit new bone formation, contributing to the reduction in bone mass observed in inflammatory bone disease [40].

\section{Conclusions}

The current study found high levels of TWEAK expression in synovial tissues and synovial fluids from patients with active RA. Together with its ability to induce cell surface expression of RANKL by osteoblasts and possibly induce activation of $\mathrm{B}$ cells into plasma cells we postulate that TWEAK in this context would be proosteoclastogenic and would thus contribute to the bone loss associated with this chronic inflammatory disease. These findings support the concept that TWEAK should be considered as a therapeutic target in RA.

\section{Abbreviations \\ ACR: American College of Rheumatology; AEC: 3,9 aminoethylcarbazole; CIA: collagen induced arthritis; DAB: diaminobenzidine; DAS28: disease activity score; DMARD: disease modifying anti-rheumatic drug; FACS: fluorescence activated cell sorting; FCS: foetal calf serum; HRP: horse raddish peroxidase; HUVEC: human umbilical vein endothelial cells; IHC: immunohistochemistry; MAb: monoclonal antibody; MAPK: mitogen-activated protein kinases; MMP matrix metalloproteinase; OA: osteoarthritis; PBMC: peripheral blood mononuclear cells; PBS: phosphate buffered saline; RA: rheumatoid arthritis; RANKL: Receptor activator of NF Kappa B Ligand; rh: recombinant human; SQA: semi-quantitative assessment; TMB: tetramethylbenzidine; TNF: Tumor necrosis factor; TRAP: tartrate resistant acid phosphatase; TWEAK: TNF-like weak inducer of apoptosis.}

\section{Acknowledgements}

This work has been supported in part by grants from the Australian National Health and Medical Research Council [ID 453568 to D.R.H]; C.J Martin Fellowship [ID299078 to T.C] and NHMRC R. Douglas Wright Fellowship to G. J.A.

The authors thank Dr. Peter Diamond, Myeloma Research Laboratory, for performing CD22 cell sorts, and Renee Ormsby, Bone Cell Biology Group, for performing real-time RT-PCR. We express our gratitude to Dale Caville, Discipline of Anatomy and Pathology, The University of Adelaide for his photographic expertise.

\section{Author details}

${ }^{1}$ Discipline of Anatomy and Pathology, School of Medical Sciences, University of Adelaide, Frome Road, Adelaide, SA 5005, Australia.

${ }^{2}$ Rheumatology Research Unit, Repatriation General Hospital, Daws Road, Adelaide, SA 5041, Australia. ${ }^{3}$ Myeloma Research Laboratory, Bone and Cancer Laboratories, Division of Haematology, Institute of Medical \& Veterinary Science, Frome Road, Adelaide, SA 5005, Australia. ${ }^{4}$ Immunology, Biogen Idec Inc., Cambridge Centre, Cambridge, MA 02142, USA. ${ }^{5}$ Bone Cell Biology Group, Discipline of Orthopaedics and Trauma, University of Adelaide, Frome Road, Adelaide, SA 5005, Australia. ${ }^{6}$ Hanson Institute, Frome Road, Adelaide, SA 5005, Australia.

\section{Authors' contributions}

AD played a major role in manuscript preparation, experimental work, statistical analysis and interpretation and MS directed patient recruitment and assessment and critical revision of the manuscript. TC carried out ELISA assays and analysis of ELISA data and wrote the manuscript. CAH and CV were substantially involved in in vitro assays. ACWZ was involved in experimental design and critical analysis of the manuscript. TSZ and DMF were involved in critical analysis of the manuscript. HMW. identified appropriate patient tissues available for the study. GJA initiated the project and was substantially involved in experimental design and critical analysis of the manuscript. DRH coordinated the project and was involved in critical revision of the manuscript.

\section{Competing interests}

The authors declare that they have no competing interests.

Received: 18 October 2010 Revised: 13 February 2011 Accepted: 24 March 2011 Published: 24 March 2011

\section{References}

1. Burkly LC, Michaelson JS, Hahm K, Jakubowski A, Zheng TS: TWEAKing tissue remodeling by a multifunctional cytokine: role of TWEAK/Fn14 pathway in health and disease. Cytokine 2007, 40:1-16.

2. Winkles JA: The TWEAK-Fn14 cytokine-receptor axis: discovery, biology and therapeutic targeting. Nat Rev Drug Discov 2008, 7:411-425. 
3. Brown SA, Richards CM, Hanscom HN, Feng SL, Winkles JA: The Fn14 cytoplasmic tail binds tumour-necrosis-factor-receptor-associated factors $1,2,3$ and 5 and mediates nuclear factor-kappaB activation. Biochem J 2003, 371:395-403

4. Campbell S, Michaelson J, Burkly L, Putterman C: The role of TWEAK/Fn14 in the pathogenesis of inflammation and systemic autoimmunity. Front Biosci 2004, 9:2273-2284.

5. Han S, Yoon K, Lee K, Kim K, Jang H, Lee NK, Hwang K, Young Lee S: TNFrelated weak inducer of apoptosis receptor, a TNF receptor superfamily member, activates NF-kappa B through TNF receptor-associated factors. Biochem Biophys Res Commun 2003, 305:789-796.

6. Campbell S, Burkly LC, Gao HX, Berman JW, Su L, Browning B, Zheng T, Schiffer $L$, Michaelson JS, Putterman C: Proinflammatory effects of TWEAK/ Fn14 interactions in glomerular mesangial cells. J Immunol 2006, 176:1889-1898.

7. Harada N, Nakayama M, Nakano H, Fukuchi Y, Yagita H, Okumura K: Proinflammatory effect of TWEAK/Fn14 interaction on human umbilical vein endothelial cells. Biochem Biophys Res Commun 2002, 299:488-493.

8. Hosokawa Y, Hosokawa I, Ozaki K, Nakae H, Matsuo T: Proinflammatory effects of tumour necrosis factor-like weak inducer of apoptosis (TWEAK) on human gingival fibroblasts. Clin Exp Immunol 2006, 146:540-549.

9. Chicheportiche Y, Chicheportiche R, Sizing I, Thompson J, Benjamin CB, Ambrose C, Dayer JM: Proinflammatory activity of TWEAK on human dermal fibroblasts and synoviocytes: blocking and enhancing effects of anti-TWEAK monoclonal antibodies. Arthritis Res 2002, 4:126-133.

10. Perper SJ, Browning B, Burkly LC, Weng S, Gao C, Giza K, Su L, Tarilonte L, Crowell T, Rajman L, Runkel L, Scott M, Atkins GJ, Findlay DM, Zheng TS, Hess H: TWEAK is a novel arthritogenic mediator. J Immunol 2006, 177:2610-2620.

11. Kamata K, Kamijo S, Nakajima A, Koyanagi A, Kurosawa H, Yagita H, Okumura K: Involvement of TNF-like weak inducer of apoptosis in the pathogenesis of collagen-induced arthritis. J Immunol 2006, 177:6433-6439.

12. Kamijo S, Nakajima A, Kamata K, Kurosawa H, Yagita H, Okumura K: Involvement of TWEAK/Fn14 interaction in the synovial inflammation of RA. Rheumatology (Oxford) 2008, 47:442-450.

13. Park MC, Jung SJ, Park YB, Lee SK: Relationship of serum TWEAK level to cytokine level, disease activity, and response to anti-TNF treatment in patients with rheumatoid arthritis. Scand J Rheumatol 2008, 37:173-178.

14. van Kuijk AW, Wijbrandts CA, Vinkenoog M, Zheng TS, Reedquist KA, Tak PP: TWEAK and its receptor Fn14 in the synovium of patients with rheumatoid arthritis compared to psoriatic arthritis and its response to TNF blockade. Ann Rheum Dis 2009, 69:301-304.

15. Xia LP, Xiao WG, Li JS, Ding S, LU J: Effects of TWEAK on the synthesis of MMP-3 in fibroblast-like synoviocytes of rheumatoid arthritis. Xi Bao Yu Fen Zi Mian Yi Xue Za Zhi 2009, 25:46-48.

16. Vincent C, Findlay DM, Welldon KJ, Wijenayaka AR, Zheng TS, Haynes DR, Fazzalari NL, Evdokiou A, Atkins GJ: Pro-inflammatory cytokines TNFrelated weak inducer of apoptosis (TWEAK) and TNFalpha induce the mitogen-activated protein kinase (MAPK)-dependent expression of sclerostin in human osteoblasts. J Bone Miner Res 2009, 24:1434-1449.

17. Polek TC, Talpaz M, Darnay BG, Spivak-Kroizman T: TWEAK mediates signal transduction and differentiation of RAW264.7 cells in the absence of Fn14/TweakR. Evidence for a second TWEAK receptor. J Biol Chem 2003, 278:32317-32323.

18. Williams SA, Martin SK, Vincent C, Gronthos S, Zheng T, Atkins GJ, Zannettino AC: Circulating levels of TWEAK correlate with bone erosion in multiple myeloma patients. Br J Haematol 2010, 150:373-376.

19. Arnett FC, Edworthy SM, Bloch DA, McShane DJ, Fries JF, Cooper NS, Healey LA, Kaplan SR, Liang MH, Luthra HS, et al: The American Rheumatism Association 1987 revised criteria for the classification of rheumatoid arthritis. Arthritis Rheum 1988, 31:315-324.

20. Altman R, Asch E, Bloch D, Bole G, Borenstein D, Brandt K, Christy W, Cooke TD, Greenwald R, Hochberg M, et al: Development of criteria for the classification and reporting of osteoarthritis. Classification of osteoarthritis of the knee. Diagnostic and Therapeutic Criteria Committee of the American Rheumatism Association. Arthritis Rheum 1986, 29:1039-1049.

21. Smith MD, Barg E, Weedon H, Papengelis V, Smeets T, Tak PP, Kraan M, Coleman M, Ahern MJ: Microarchitecture and protective mechanisms in synovial tissue from clinically and arthroscopically normal knee joints. Ann Rheum Dis 2003, 62:303-307.

22. Kraan MC, Haringman JJ, Post WJ, Versendaal J, Breedveld FC, Tak PP: Immunohistological analysis of synovial tissue for differential diagnosis in early arthritis. Rheumatology (Oxford) 1999, 38:1074-1080.

23. Dharmapatni AA, Smith MD, Findlay DM, Holding CA, Evdokiou A, Ahern MJ, Weedon $\mathrm{H}$, Chen P, Screaton G, Xu XN, Haynes DR: Elevated expression of caspase-3 inhibitors, survivin and XIAP correlates with low levels of apoptosis in active rheumatoid synovium. Arthritis Res Ther 2009, 11:R13.

24. Gomori E, Pal J, Abraham H, Vajda Z, Sulyok E, Seress L, Doczi T: Fetal development of membrane water channel proteins aquaporin-1 and aquaporin-4 in the human brain. Int J Dev Neurosci 2006, 24:295-305.

25. Atkins GJ, Kostakis P, Vincent C, Farrugia AN, Houchins JP, Findlay DM, Evdokiou A, Zannettino AC: RANK expression as a cell surface marker of human osteoclast precursors in peripheral blood, bone marrow, and giant cell tumors of bone. J Bone Miner Res 2006, 21:1339-1349.

26. Vincent C, Kogawa M, Findlay DM, Atkins GJ: The generation of osteoclasts from RAW 264.7 precursors in defined, serum-free conditions. J Bone Miner Metab 2009, 27:114-119.

27. Simmons PJ, Torok-Storb B: Identification of stromal cell precursors in human bone marrow by a novel monoclonal antibody, STRO-1. Blood 1991, 78:55-62.

28. Atkins GJ, Welldon KJ, Halbout P, Findlay DM: Strontium ranelate treatment of human primary osteoblasts promotes an osteocyte-like phenotype while eliciting an osteoprotegerin response. Osteoporos Int 2008, 20:653-664.

29. Nitschke L: The role of CD22 and other inhibitory co-receptors in B-cell activation. Current Opinion in Immunology 2005, 17:290.

30. Dorner T, Goldenberg DM: Targeting CD22 as a strategy for treating systemic autoimmune diseases. Ther Clin Risk Manag 2007, 3:953-959.

31. Haynes DR, Crotti TN, Loric M, Bain Gl, Atkins GJ, Findlay DM: Osteoprotegerin and receptor activator of nuclear factor kappaB ligand (RANKL) regulate osteoclast formation by cells in the human rheumatoid arthritic joint. Rheumatology (Oxford) 2001, 40:623-630.

32. Kataria N, Bartold PM, Dharmapatni AASK: Expression of tumor necrosis factor-like weak inducer of apoptosis (TWEAK) and its receptor, fibroblast growth factor-inducible 14 protein (Fn14), in healthy tissues and tissues affected by periodontitis. J Periodontal Res 2010, 45:564-573.

33. Lynch CN, Wang YC, Lund JK, Chen YW, Leal JA, Wiley SR: TWEAK induces angiogenesis and proliferation of endothelial cells. J Biol Chem 1999, 274:8455-8459.

34. Donohue PJ, Richards CM, Brown SA, Hanscom HN, Buschman J, Thangada S, Hla T, Williams MS, Winkles JA: TWEAK is an endothelial cell growth and chemotactic factor that also potentiates FGF-2 and VEGF-A mitogenic activity. Arterioscler Thromb Vasc Biol 2003, 23:594-600.

35. Nicholson GC, Malakelis M, Collier FM, Cameron PU, Holloway W, Gough TJ, Gregorio-King C, Kirkland MA, Myers DE: Induction of osteoclast from CD14-positive human peripheral blood mononuclear cells by activator of nuclear factor kappaB ligand (RANKL). Clin Sci (Lond) 2000, 99:133-140.

36. Atkins GJ, Kostakis P, Welldon KJ, Vincent C, Findlay DM, Zannettino AC: Human trabecular bone-derived osteoblasts support human osteoclast formation in vitro in a defined, serum-free medium. J Cell Physiol 2005, 203:573-582.

37. Atkins GJ, Kostakis P, Pan B, Farrugia A, Gronthos S, Evdokiou A, Harrison K, Findlay DM, Zannettino AC: RANKL expression is related to the differentiation state of human osteoblasts. J Bone Miner Res 2003, 18:1088-1098.

38. Paszty $\mathrm{C}$, Turner $\mathrm{CH}$, Robinson MK: Sclerostin: a gem from the genome leads to bone-building antibodies. J Bone Miner Res 2010, 25:1897-1904.

39. Atkins GJ, Rowe PS, Lim HP, Welldon KJ, Ormsby RT, Wijenayaka AR, Zelenchuk L, Evdokiou A, Findlay DM: Sclerostin is a locally acting regulator of late-osteoblast/pre-osteocyte differentiation and regulates mineralization through a MEPE-ASARM dependent mechanism. J Bone Miner Res 2011.

40. Walsh NC, Reinwald S, Manning CA, Condon KW, Iwata K, Burr DB, Gravallese EM: Osteoblast function is compromised at sites of focal bone erosion in inflammatory arthritis. J Bone Miner Res 2009, 24:1572-1585.

doi:10.1186/ar3294

Cite this article as: Dharmapatni et al:: TWEAK and Fn14 expression in the pathogenesis of joint inflammation and bone erosion in rheumatoid arthritis. Arthritis Research \& Therapy 2011 13:R51. 